\title{
SOME FACTORS INFLUENCING THE SPREAD OF SCARLET FEVER IN AN INSTITUTION
}

By E. V. KEOGH, M.B., IAN MACDONALD, M.B., JOAN BATTLE, B.Sc., R. T. SIMONS, A.A.C.I. AND STANLEY WILLIAMS, M.D.

\section{From the Commonwealth Serum Laboratories, Melbourne}

\section{INTRODUCTION}

IN the admirable pioneer studies of Griffith and his colleagues on the epidemiology of streptococcal infections in schools, "no systematic attempt was made to discover the distribution of streptococci amongst the healthy residents. And the observations on carriers were in general confined to those who were suspected to have some catarrhal infection of the upper air passages" (Griffith, 1938). In this paper we report the results of weekly swabbings of the throats of all children in a single dormitory or ward of an orphanage with 350 inmates, during an outbreak of scarlet fever due to Streptococcus pyogenes, serologically type 2 (Griffith). All haemolytic streptococci were typed by the slide agglutination technique, so that fluctuations in the distribution of the epidemic and non-epidemic strains could be followed.

\section{HISTORY OF THE EPIDEMIC}

Our department was called in to investigate the epidemic some time after it began, and we restricted our work to a single ward. The children in this ward were all girls, from 4 to 17 years old, the average age being 8 years. Only two girls were more than 14 years of age. The ward normally contained sixty children. Type 2 was isolated from every case of scarlet fever which arose in the institution. This type was found to be responsible for $50 \%$ of cases of scarlet fever occurring in the city of Melbourne during the period April to September 1938. During these months the disease had not assumed unusual proportions, and the cases occurred as follows:

\begin{tabular}{|c|c|c|}
\hline \multicolumn{2}{|c|}{12 September 1938} & \multirow{2}{*}{$\begin{array}{l}3 \text { cases ( } 1 \text { death-the only death in the outbreak). } \\
4 \text { cases. }\end{array}$} \\
\hline 13 & , & \\
\hline 14 & 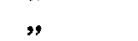 & 1 case. \\
\hline 29 & " & 2 cases. \\
\hline & ber 1938 & 1 case. \\
\hline 22 & , & 2 cases. \\
\hline 23 & ", & 1 case. \\
\hline 27 &, & 1 case. \\
\hline
\end{tabular}

None of the September cases, but all of those in the last week of October, arose in the ward with which we are concerned, and the subsequent course of events is the subject of this paper. The course of the epidemic in the other wards of the institution will not be discussed. 
On 28 October throat swabs were taken from the fifty-nine children in this ward. Children were not admitted to the ward during the nine weeks of our observations. Those developing scarlet fever were sent to a fever hospital as soon as the diagnosis was made. The children in the ward were isolated from direct contact with other children in the institution, but there was limited indirect contact through some members of the staff. The ward consisted of nineteen rooms, five containing single beds, and the rest four beds each. There was a separate playroom and playground common to the occupants of the ward. Children carrying type 2 were strictly isolated in rooms in the ward. The findings should not be regarded as illustrating the unhampered spread in a semi-closed community of the epidemic strain, as efforts were made to arrest the spread of infection by isolation of carriers within the ward and by treatment with sulphanilamide. The possible effects of these measures will be discussed later. Although members of the staff were swabbed occasionally, we were unfortunately unable to arrange for their systematic examination.

\section{Methods}

Swabs of the throats of all the children in the ward were taken by one of us, who at the same time noted the clinical condition of the children, in particular the appearance of the throat and tonsils. The swabs were rubbed firmly over the surfaces of both tonsils. No attempt was made to express secretions from the crypts. These swabs were plated the same day on neopeptone horse blood agar (Ward \& Rudd, 1938) and incubated overnight at $37^{\circ} \mathrm{C}$. The following day the number of colonies of beta haemolytic streptococci on each plate was noted and all examined with a plate microscope $(x 16)$ by reflected and transmitted light. The types of colonies were very constant and a single colony was sub-cultured into tryptic digest broth and incubated overnight at $30^{\circ} \mathrm{C}$. Subcultures on blood agar were made from the broth cultures, the deposit from which was used for typing by Griffith's slide agglutination method. If the strain could not be typed by the group A type sera at our disposal the Lancefield group was determined, using the formamide method of Fuller (1938) for extraction of the group polysaccharide. During the course of the investigation it was found that this procedure was liable to error, since apparently specific agglutination of non-A strains sometimes occurred with our type 4 or type 28 sera. It was, therefore, necessary to check the group of any strain agglutinating with either of these sera. As samples of these type sera from other laboratories also agglutinated these non-A strains, we have modified our routine procedure. All strains received at this laboratory are now, prior to typing, subjected to the presumptive cultural test of Ward \& Rudd (1938) for identification of group A streptococci. One of us has published elsewhere his satisfactory experience with this simple test (Keogh, 1939). The sera used were prepared from the type strains generously supplied by Dr F. Griffith. Following the procedure of Pauli \& Coburn (1937), they were absorbed with group A polysaccharide prepared by the method of Fuller. As this method in our hands did 
not render the sera completely type specific, they were subsequently absorbed with the strains of other types which they agglutinated non-specifically. The sera had already been used to type large numbers of locally isolated strains (Keogh \& Kelsey, 1939).

\section{Results}

There were ten consecutive weekly swabbings of the children, followed by another swabbing after an interval of 13 weeks. The results are shown in Table I. Haemolytic streptococci were isolated on one or more occasions from the throats of fifty of the fifty-four children examined. The epidemic strain, type 2, was recovered at least once from thirty-four of fifty-four children during the first 9 weeks. It was also responsible for the five cases of scarlet fever which arose in the week prior to the first swabbing, so that it was isolated from thirtynine of fifty-nine children.

Nine children developed scarlet fever in the first month of the observation period, and two more in the interval of 13 weeks between the penultimate and final swabbings, when the isolation restrictions were relaxed. These restrictions were removed as no cases of scarlet fever had occurred in the preceding 5 weeks, and the routine of the institution made any contact with children from other wards merely accidental and casual. Of the thirty-nine children in whom type 2 was detected, sixteen developed scarlet fever and twenty-three did not. It is improbable that we detected every child who carried type 2 at any time, but an incidence of sixteen attacks of scarlet fever in a ward containing fifty-nine children indicates the high virulence of the epidemic strain. Dick tests were carried out too late to provide any useful information in lack of knowledge of the pre-epidemic Dick status. Practically all the children from whom type 2 was recovered in the first month showed clinical signs of nasopharyngitis, but similar signs and symptoms were noted in many of the children from whom we failed to isolate it.

Type 2 was never isolated from any child, who developed scarlet fever, before the appearance of the rash, indicating a very short incubation period. Five children admitted to other wards of the institution developed scarlet fever within $48 \mathrm{hr}$. of admission.

The numbers of type 2 colonies present on the plates of positive swabs steadily diminished during the period of observation (Table II). A decrease in the numbers of streptococci recoverable from individual throats usually took place before their complete disappearance (Table I). Failure to recover streptococci did not necessarily imply that they were no longer present in the tonsils, since type 2 was recovered in large numbers from the tonsils removed from two children who, after a series of positive swabs, had been consistently negative. An analysis of the results of the swabbings from the twenty-three children positive for type 2 showed that, on thirteen occasions, two consecutive swabs were positive; on nine occasions, one negative swab intervened between two positives; on six occasions two negatives intervened, and on a further six. 


\section{Table I. The types of streptococci in the throats of fifty-three children} in one ward during a scarlet-fever epidemic

Weekly swabs: 28 October-28 December

28 March

(interval 13 weeks)

Eleven cases of scarlet fever, due to type 2

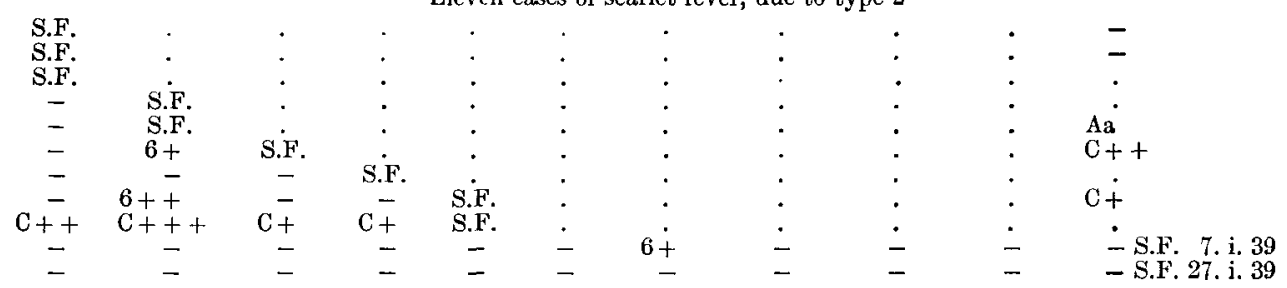

Twenty-three type 2 infections without rash

\begin{tabular}{|c|c|c|c|c|c|c|c|c|c|c|}
\hline $2+++$ & $-2++$ & $\mathrm{C}+++$ & - & $2++$ & - & $\mathrm{C}+$ & $\mathrm{C}+++$ & $\mathrm{C}+++$ & $\mathrm{C}+$ & $\mathrm{C}+++$ \\
\hline $2++$ & - & - & - & $2++$ & - & $2++$ & $2+$ & - & $\mathrm{Aa}+++$ & - \\
\hline $2+++$ & - & $2++$ & - & $2++$ & - & - & - & - & $\mathrm{C}+++$ & . \\
\hline $\begin{array}{l}2++t \\
2++t\end{array}$ & - & - & - & $2++$ & - & $2+$ & $2+-$ & $2+$ & $\begin{array}{l}2+ \\
2+\end{array}$ & $\mathbf{C}+$ \\
\hline $2+t+$ & - & - & - & $2++$ & $2++$ & $2+$ & $2+$ & - & - & - \\
\hline $2++$ & - & - & - & - & _- & - & $\mathbf{A a}++$ & - & - & . \\
\hline $2+$ & - & - & - & - & - & - & - & - & $\mathrm{Aa}++$ & - \\
\hline $2+++$ & - & $4+$ & - & $2++$ & - & - & - & - & - & $\mathrm{C}++$ \\
\hline $2++$ & - & - & - & & & $\cdot$ & & ${ }^{\circ}$ & - & - \\
\hline $2+++$ & - & - & - & $2+$ & - & $2+$ & - & $2++$ & - & - \\
\hline $2+++$ & - & - & - & - & - & - & - & - & $2+$ & - \\
\hline $2++$ & - & $A c++$ & $\mathrm{Ac}++$ & $A c++$ & - & - & $A c+$ & - & - & - \\
\hline- & $2++$ & - & - & $2++$ & - & - & - & - & - & . \\
\hline *- & - & $2++$ & - & $2++$ & - & - & - & - & - & . \\
\hline - & $\mathrm{C}+++$ & $2++$ & $2++$ & $2++$ & $2+$ & $2+$ & $2+$ & $2+$ & - & \\
\hline & - & - & - & $2+$ & - & - & - & - & - & $\mathrm{C}++$ \\
\hline *27+ & - & - & - & $2+$ & $2+$ & - & - & - & - & - \\
\hline- & - & - & - & $2+$ & - & - & $2+4$ & - & - & - \\
\hline$?+$ & - & - & - & $2++$ & -- & $2++$ & - & - & $2++$ & - \\
\hline - & $\mathrm{Ae}++$ & - & - & $\mathrm{Ae}++$ & $2++$ & - & - & - & - & . \\
\hline *- & - & - & - & - & - & - & $2++$ & - & - & - \\
\hline * - & - & - & - & - & - & - & - & - & $2+$ & . \\
\hline & & & & hirteen $\mathrm{c}$ & hildren i & $\mathrm{ed} w$ & ith types of & than & & \\
\hline $6+++$ & $-6+$ & $6++$ & $6++$ & $6++$ & $6++$ & $6+++$ & $6+++$ & $6++$ & - & $\mathrm{C}++$ \\
\hline $6++$ & $6++$ & - & - & $6++$ & $6+$ & $6+$ & - & $6+$ & - & - \\
\hline *- & - & $6+-$ & - & - & - & - & - & - & - & $\mathrm{C}++$ \\
\hline - & - & ${ }^{+}$ & - & $6+$ & - & $\overline{-}$ & $\overline{-}$ & $\overline{-}$ & I & - \\
\hline $\mathrm{C}++$ & $\mathrm{C}+$ & & & & & & & & . & : \\
\hline- & - & $\mathrm{C}++$ & - & $\mathrm{C}++$ & $\mathrm{C}++$ & ++ & $\mathrm{C}++$ & $\mathrm{C}++$ & $\mathrm{C}+$ & \\
\hline $\mathrm{C}++$ & $\mathrm{C}++$ & - & - & $27++$ & $27++$ & $27++$ & $\mathrm{C}+++$ & $\mathrm{C}++$ & - & $\mathrm{Ad}+$ \\
\hline - & - & - & - & $\mathrm{C}++$ & - & $1+\overline{+}$ & $4+\bar{t}$ & - & $\mathrm{C}_{+}{ }^{\circ}$ & - \\
\hline *_- & - & - & - & - & - & - & $4+$ & $\mathrm{Aa}+-$ & ${ }^{+}$ & - \\
\hline- & + & - & - & $\mathrm{Ad}++$ & $d+$ & $\mathrm{Ad}++$ & $\mathrm{Ad}++$ & - & $\mathrm{Ad}+$ & $\mathrm{C}++$ \\
\hline $1 b+++$ & - & - & - & $A b+$ & - & $A b++$ & $\mathrm{Ab}++$ & $\mathrm{Ab}++$ & $\mathrm{Ab}++$ & - \\
\hline
\end{tabular}

Seven children who gave negative swabs, except three at the final swabbing

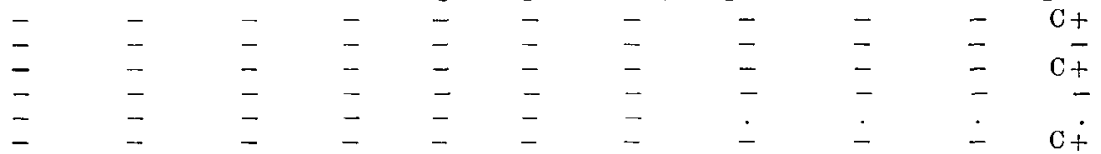

S.F. $=$ scarlet fever.

The numbers indicate the serological type of the streptococcus found: $\mathrm{Aa}=$ type "Stewart"; $\mathrm{Ab}=$ "Hale"; $\mathrm{Ac}=$ "Co $\mathrm{h}$ lan"; Ad = "Jackson"; Ae="Alli".

The crosses indicate the numbers of colonies of haemolytic streptococci on the plates: $++t=0$ ver 100 colonies: $++=100-10$ colonies; $+=10$ colonies; $-=$ no colonies; $\cdot=$ no swab taken

* Indicates the children without tonsils 


\section{Table II. Diminution of numbers of streptococci recoverable} from infected throats during the epidemic

$\begin{array}{cc}\text { Colonies per plate } & \text { 1st week } \\ \text { Over } 100 & 11 \\ >10<100 & 4 \\ <10 & 2 \\ \text { Numbers of new } & 16 \\ \text { positives }\end{array}$

$\begin{array}{cc}\text { 2nd three } & \text { 3rd five } \\ \text { weeks } & \text { weeks } \\ 0 & 1 \\ 17 & 7 \\ 7 & 14 \\ 7 & 3\end{array}$

occasions three negatives. In one child only did more than three negatives occur between positives. In this case eight negatives were obtained between the first and second positive swabs. It is evident that a child could not be considered free from infection of the surface of the tonsils until three consecutive negative swabs had been obtained. Possibly the child who gave a second positive after eight successive negative swabs had been reinfected with type 2.

Eight other types of haemolytic streptococci were encountered, seven belonging to group A, and one to group C. The group A strains were types 4, 6, 27, "Stewart" and "Alli" (the latter two are locally identified types not yet included in Griffith's series), and two strains "Coghlan" and "Jackson", found only in the two children of those names, who were persistent carriers of them.

Types 4, 6, 27, "Stewart" and "Alli" are the types most frequently found in Melbourne in tonsils removed at operation, but are isolated from scarletfever patients only on rare occasions. There were two persistent carriers of type 6. Type 6 was also isolated from three out of eight children who subsequently developed scarlet fever, from whom we had obtained at least one swab before infection with type 2. It was not isolated from any of the twentythree children infected with type 2 who did not develop a rash, though some had suffered from sore throat and other clinical signs, such as reddening of the faucial pillars. This difference in incidence of type 6 infections in the two groups is significant $(P=0 \cdot 0124)$. It appears that, as a group, children, possessing sufficient antitoxic immunity to prevent the appearance of a rash following infection with the potent scarlatinal type 2 strain, had also sufficient antibacterial immunity to resist completely infection with type 6 . Since eight of these twenty-three children were, at one time or another, infected with other group A strains, their resistance to type 6 infection was presumably type specific. The presence in the ward of two persistent carriers of type 6 suggests to us that, prior to our observations, type 6 may have been spreading freely, and that children, who had contracted a type 6 infection and recovered from it, had developed sufficient antitoxin to prevent a rash following a type 2 infection, and were also immune to type 6 by reason of the previous infection with that strain. Those, on the other hand, who had only recently been infected, that is, in the period of our observations, had presumably not had sufficient time to develop sufficient antitoxin to suppress a rash when infected by type 2 . We do not imply that all the children who contracted type 2 infec- 
tion without rash owed their resistance to prior type 6 infection, but that a sufficient number of them did so to account for the discrepancy in incidence of type 6 infections in the two groups (type 2 infections with and without rash). Many of the group may have been immunized by one or more of the other "non-scarlatinal" group A strains and not have been exposed to an infecting dose of type $6 .{ }^{1}$

This speculative interpretation assumes that, in the throats of children, the type 6 strain is only weakly toxigenic, not producing sufficient toxin in a short enough time to provoke a rash in children of average susceptibility, but producing the requisite amount of toxin to stimulate the slow development of antitoxin. We have isolated type 6 from scarlet-fever patients in 3 out of 196 isolations, and White et al. (1939) isolated it in 11 out of 315 isolations, indicating that type 6 infection is, in fact, capable of producing a rash in an occasional child. We have not tested it for in vitro production of toxin, since a negative result would not negative the above hypothesis. It seems not unlikely that infection with weakly toxigenic strains may be largely responsible for the development of scarlatinal antitoxin in the human being. The possible alternative process of sub-clinical infection with potent scarlatinal strains seems less probable.

It has been suggested to us that the above facts are susceptible to other interpretations, of which the simplest is to assume that the epidemic strain (type 2), if implanted in a throat, ousts or prevents the implantation of less virulent strains. Such a process, while it probably occurs, can hardly account for the facts in this series of observations. For, of the twenty-three type 2 infections without rash, seven children carried A or C strains, other than type 6, subsequently to type 2 infection, and four prior to infection. This total attack rate by strains other than types 2 or $6(11 / 23)$ is almost identical with that $(14 / 27)$ in the remaining children. Rather, the absence of type 6 from the throats of these children is entitled to more significance than is deduced from the crude attack rates, since they were in contact with the type 6 carriers over a longer period, with consequent increased risk of infection, and were also swabbed on more occasions (average ten times as compared to five), increasing the chance of detecting type 6 in their throats.

We have further tried to eliminate this factor by considering only the cases from whom we had obtained at least one swab before type 2 infection, with or without rash. In the children who developed scarlet fever there were three swabs (from three separate children) positive for type 6 of twenty-six taken, in those infected with type 2 , without rash, there were fifty-two negative swabs. The probability that this difference is not due to chance is 0.034 , which reaches the accepted level of significance. This argument may seem to be unduly laboured; but it illustrates the difficulty of assessing the significance of data obtained in this type of enquiry. And since field observations on large numbers

1 The probabilities in this section, involving numbers less than five in some classes, have been calculated on the basis of absolute probability, and not by reference to tables of $X^{2}$. 
of children are laborious and time-consuming, it is desirable that all the relevant information possible should be extracted from the results.

The group $\mathrm{C}$ strains were all agglutinated by a serum prepared from one of them, which was absorbed by group $\mathrm{C}$ polysaccharide. The serum did not agglutinate the Griffith strains belonging to group $\mathrm{C}$ (types 7, 20, 21), and only agglutinated a few of the group $\mathrm{C}$ strains isolated from other sources in Melbourne. This group C strain was encountered throughout the period of observation, and at the final swabbing was recovered from the throats of twelve of thirty-three children, of whom only one carried a group A strain (type "Stewart"). Despite its high infectivity, there was no evidence that this C strain gave rise to any clinical symptoms.

\section{THE EFFECT OF TONSILLECTOMY}

Of the fifty-four children, nine had had their tonsils removed and five of the nine were infected by type 2 , as compared with seventeen of thirty-four children with tonsils. Similarly, four of the nine tonsillectomized children were infected with strains other than type 2, as compared with twenty of the thirtyfour children with tonsils. The children without tonsils, therefore, had no diminished susceptibility to streptococcal infection, but the infection, once contracted, persisted much longer in children with tonsils. Out of a total of thirty-five individual swabs taken, following the initial infection, from the tonsillar fossae of six children without tonsils, four were positive; twenty-eight children with tonsils gave eighty-four positive swabs in 201 such swabbings. This difference is significant $(P<0 \cdot 001)$. As a very rough approximation, it may be stated that the streptococci were, on the average, recoverable for 1-2 weeks from the tonsillectomized child, and for 4-5 weeks from the children with tonsils.

\section{THE EFFECT OF SULPHANILAMIDE THERAPY}

Two attempts were made to arrest the outbreak by sulphanilamide therapy. From 4-8 November, inclusive, all the children and three members of the staff from whom type 2 had been isolated at the initial examination on 28 October, were given "Proseptasine" $(0.5 \mathrm{~g}$. to the children, and $1.0 \mathrm{~g}$. to the adults three times daily). This measure failed to eliminate the type 2 infection. From 14-20 November all the inmates of the ward, including the staff, were given Proseptasine in the doses mentioned. Two children developed scarlet fever while on sulphanilamide, one on the second and one on the fifth day of treatment. More children were carrying type 2 on 24 November, 4 days after the drug was stopped, than at any other period during the epidemic. We conclude that the preparation we used, in the amounts and dose-intervals employed, had no demonstrable prophylactic effect against infection with the type 2 strain responsible for the epidemic. 


\section{THE EFFECT OF ISOLATION}

Following the results of the first swabbing, an attempt was made to isolate known carriers of type 2 from the other children. The ward was divided into sufficient small rooms to make this possible. Several carriers were put together into one room. As it was 2 days before the result of a weekly swabbing was known, type 2 carriers had ample opportunity of passing on the infection before they were detected. In these circumstances it is not surprising that the isolation measures had very little apparent effect early in the epidemic. But, in the later stages, when carriers were excreting streptococci in smaller numbers, as shown by the relatively sparse growth on the plates, these same measures did appear to be a substantial obstacle to spread of the epidemic strain, since no new cases of scarlet fever occurred in the last 5 weeks. This was due neither to the absence of susceptible children nor to a loss of pathogenicity of the type 2 strain in the throats of carriers, for when the restrictions were relaxed, two of the children developed scarlet fever, while cases of scarlet fever, which could be attributed only to casual contact with the released type 2 carriers, occurred in children from other wards.

\section{Discussion}

The results of this investigation confirm the previous impression (Keogh \& Kelsey, 1939) that in Melbourne at the present time certain strains of streptococci can be regarded as "scarlatinal" and other strains as "non-scarlatinal". In Melbourne types 2 and 17 are isolated from the great majority of scarletfever patients and only rarely from other sources, such as infected tonsils or surgical infections, in which types 6 and "Stewart" are common. Of these types present in the outbreak under discussion, type 2 was responsible for all the cases of scarlet fever, although types 6 and "Stewart" were spreading freely in the ward. We do not think that the type 2 strain is characterized by greater infectivity (as distinct from virulence or toxigenicity) in comparison with the other strains. Although more children were infected with type 2 than with either type 6 or "Stewart", there is suggestive evidence that many may have been specifically immune to infection with type 6 , owing to prior infection. Type 2 was, on the contrary, a fresh introduction. The group $\mathrm{C}$ strain, which, as far as we could determine, was non-pathogenic, was highly infectious, being recovered at least once from twenty children. It seems impossible to give a satisfactory definition of infectivity as an isolated property of a strain, since the apparent infectivity of a strain must be influenced by many imponderable factors, e.g. the immunological history of the exposed community, the ability of the strain to multiply rapidly or to maintain itself for long periods in an infected throat, or to resist destruction in air or dust when excreted.

White et al. (1939) have shown that capsulated strains have a greater infectivity than non-capsulated strains, since the incidence of familial infections is significantly higher with capsulated strains. They are "inclined to think that the tendency of capsulated strains to spread, often causing a little 
epidemic of scarlet fever in the family and occasionally a larger epidemic in the community, is due both to their greater infectivity and to their greater toxigenic capacity". They regard "infectivity" as a property distinct from virulence or toxigenic capacity. We do not entirely agree with this interpretation of their very interesting observation.

We would define a scarlatinal strain as one which can produce with suffcient rapidity, i.e., within the short incubation period, enough erythrogenic toxin to determine the appearance of a rash in the great majority of Dicknegative children (cf. Topley \& Wilson, 1936). This power to produce sufficient toxin may depend on (1) the ability of the strain to multiply rapidly in the throat, or on (2) the inherent toxigenicity of the strain. There may, of course, be a high correlation between these properties. Capsulation is not, we think, correlated with inherent high toxigenicity, because though types 2 and 6 are both capsulated strains, one is commonly associated with scarlet fever, the other only rarely. Type "Wade", the epidemic strain in Adelaide at present (Keogh et al. 1939), is non-capsulated. We think it more likely that capsulation, by rendering the strain resistant to the natural defences of the host, favours the rapid attainment and maintenance of a high concentration of the cocci in the throat. Such a process would render a strain apparently more infectious, since it would produce a higher average infecting dose in the patient's discharges of organisms which would, ex hypothesi, also resist the natural defences of the contact.

The possibility exists that strains of varying infectivity and toxigenicity may exist within the one serological type. We have isolated both capsulated and non-capsulated strains of type 12 from different patients. Type 6 , which is rarely associated with scarlet fever in England and Australia, has been isolated in outbreaks of scarlet fever in America (Bailey, 1939).

The Schools' Epidemics Committee of the Medical Research Council, studying the incidence of nasopharyngeal infections in boys and girls with and without tonsils, found no significant difference in the attack rates on the two groups (Schools' Epidemics Committee, 1938). Our very limited observations agree as far as streptococcal infections are concerned, but we find that the streptococcal infection, once contracted, persists very much longer in children with tonsils. This conclusion is based on the examination of a few children only, but is supported by so much clinical evidence that it may appear platitudinous. If, as we believe, the tonsils are the chief reservoir of group A streptococci, wholesale tonsillectomy, were it practicable, might greatly reduce, if not abolish, human streptococcal disease. On a less exalted plane, we feel justified in concluding that the presence in an institution of a large proportion of children with tonsils is a factor favouring the continuance of an epidemic of scarlet fever. Conversely, the presence in the English public schools of a very high proportion of tonsillectomized children (50\%) may have been partly responsible for the surprising inability of scarlet fever to attain epidemic proportions in them (Schools' Epidemics Committee, 1938). 


\section{SUMMARY}

1. An epidemic of scarlet fever due to Streptococcus pyogenes, type 2 (Griffith), in a ward of fifty-nine children is described. The children were swabbed weekly for 10 weeks, and again, once, after an interval of 13 weeks.

2. All haemolytic streptococci isolated were examined serologically. Sixteen children developed scarlet fever (type 2 infection with rash), twenty-three contracted type 2 infection without rash. Haemolytic streptococci were isolated at least once from all but four children.

3. Seven group A strains and one group $\mathrm{C}$ strain were present in the ward. Two group A strains and the group C strain spread freely, but were not associated with scarlet fever, or any evident clinical symptoms.

4. There is suggestive evidence that a number of the children, who failed to develop a rash following infection with type 2 , owed their resistance to a previous infection by type 6 without rash.

5. Children with and without tonsils were equally susceptible to infection with haemolytic streptococci, irrespective of group or type, but the infections persisted much longer in the children with tonsils.

6. Sulphanilamide therapy did not check the epidemic.

\section{REFERENCES}

BAILEY, J. H. (1939). Amer. J. Hyg. B, 29, 107.

Fuller, A. T. (1938). Brit. J. exp. Path. 19, 130.

GRIFFITH, F. (1938). Spec. Rep. Ser. med. Res. Coun., Lond., no. 227, p. 267.

KеоGн, E. V. (1938). Med. J. Aust. 2, 1057.

KmoGн, E. V. \& KeLSEY, H. (1939). Med. J. Aust. 1, 100.

Keogh, E. V., Macdonald, I., Battle, J. \& Puckey, M. (1939). Med. J. Aust. 1, 792.

PAULI, R. H. \& CoBdrn, A. F. (1937). J. exp. Med. 65, 595.

Schools' Epidemics Committee (1938). Spec. Rep. Ser. med. Res. Coun., Lond., no. 227, p. 164.

Topley, W. W. C. \& WILson, G. F. (1936). The Principles of Bacteriology and Immunity. Second edition, p. 1169.

WARD, H. K. \& RUDD, G. V. (1938). Aust. J. Exp. Biol. med. Sci. 16, 181.

White, C., Rudd, G. V., Ward, H. K., Wilson, F. H. \& Symington, N. J. (1939). Med. J. Aust. 1, 96.

(MS. received for publication 9. vIII. 39-Ed.) 